\title{
Coronary artery anomalies and their clinical relevance
}

\section{Le anomalie coronariche e la loro rilevanza clinica}

\author{
Leonardo Misuraca, Giovanni Benedetti, Anna Sonia Petronio, Marco De Carlo, \\ Piersilvio Chella, Andrea Pieroni, Alberto Balbarini
}

\begin{abstract}
Coronary artery anomalies and their clinical relevance. L. Misuraca, G. Benedetti, A.S. Petronio, M. De Carlo, P. Chella, A. Pieroni, A. Balbarini.

Coronary artery anomalies (CAAs) represent one of the most confusing topic in cardiology and affect approximately $1 \%$ of the general population. Altough some anomalies seem to be only anatomical curiosities, others may sometimes have fatal consequences. This review describes
\end{abstract}

\begin{abstract}
the anatomical characteristics of main CAAs and focuses on the pathophysiological mechanisms by which CAAs may cause a pathological state. The last section describes these therapeutical options of this congenital disorders. raphy.

Keywords: coronary artery anomalies, coronary angiog-
\end{abstract}

Monaldi Arch Chest Dis 2011; 76: 66-71.

Cardiac catheterization laboratory, Cardio-Thoracic and Vascular Department, Azienda Ospedaliero-Universitaria Pisana, Pisa, Italy.

Corresponding author: Leonardo Misuraca;+39050995330; E-mail address:leo.misuraca@gmail.com

According to literature, coronary artery anomalies (CAAs) affect approximately $1 \%$ of the general population; this percentage is derived from cineangiograms performed for suspected obstructive disease [1-2].

Angelini and coworkers [2] propose that, because of its substantial variability, normal and anomalous coronary anatomy should be well defined. Coronary anatomy is normal when it is observed in $>1 \%$ of an unselected population; a normal variant is an alternative, relatively unusual, morphological feature seen in $>1 \%$ of the same population; and anomaly, a morphological feature seen in $<1 \%$ of that population. The incidence of coronary anomalies is relevant not only for conceptual and educational purposes but, more importantly, for public health issues. In fact, $19 \%$ of sudden deaths in young athletes in USA are related to these anomalies [3].

An accepted classification of CAAs is the following [2] (Table 1):

1) Anomalies of origination and course, including cases of absence of left main trunk (split origination of LCA, Figure 1.), anomalous location of a coronary ostium outside aortic sinuses, for example from ascending aorta or pulmonary artery, or other anomalous coronary patterns at risk (Fig. 2).
2) Anomalies of intrinsic coronary arterial anatomy: relatively frequent are coronary hypoplasia or intramural coronary artery (muscle bridge), split RCA or split LAD, double LAD and LAD with a large septal branch. Hypoplastic coronary arteries have a small luminal diameter (usually less than $1 \mathrm{~mm}$ ) and a reduced length. Rare patterns include congenital atresia or stenosis or the absence of a coronary artery. Congenital epicardial coronary stenosis is usually caused by a membrane or a fibrotic ridge. Coronary atresia is characterized by the presence of an ostial dimple in the left or right aortic sinus that terminates in a cordlike fibrotic structure without a patent lumen. In some individuals, certain
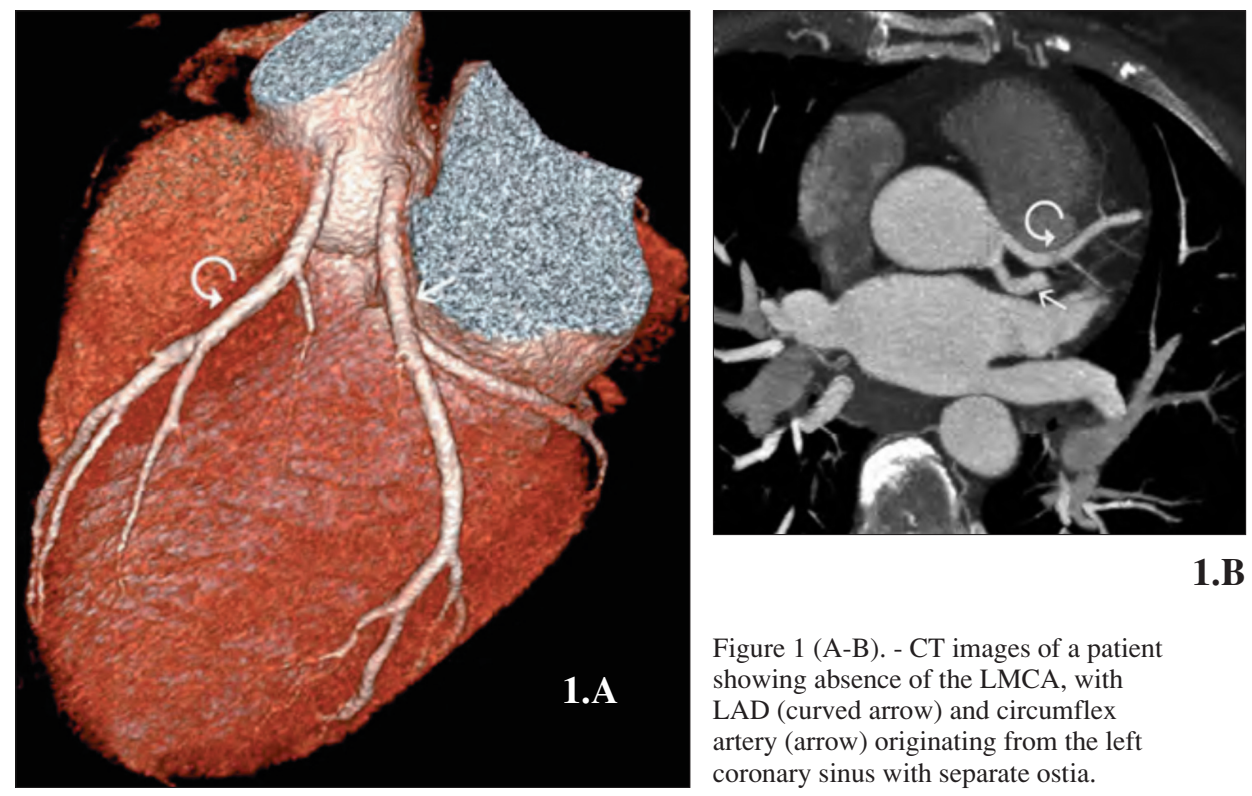

1.B

Figure 1 (A-B). - CT images of a patient showing absence of the LMCA, with LAD (curved arrow) and circumflex artery (arrow) originating from the left coronary sinus with separate ostia. 
Table 1. - Classification of Coronary Anomalies in Human Hearts

\section{A. Anomalies of origination and course}

1. Absent left main trunk (split origination of LCA)

2. Anomalous location of coronary ostium within aortic root or near proper aortic sinus of Valsalva (for each artery)
a. High
b. Low
c. Commissural

3. Anomalous location of coronary ostium outside normal "coronary" aortic sinuses

a. Right posterior aortic sinus

b. Ascending aorta

c. Left ventricle

d. Right ventricle

e. Pulmonary artery

(1) LCA that arises from posterior facing sinus

(2) $\mathrm{Cx}$ that arises from posterior facing sinus

(3) LAD that arises from posterior facing sinus

(4) RCA that arises from anterior right facing sinus

(5) Ectopic location (outside facing sinuses) of any coronary artery from pulmonary artery

(a) From anterior left sinus

(b) From pulmonary trunk

(c) From pulmonary branch

f. Aortic arch

g. Innominate artery

h. Right carotid artery

i. Internal mammary artery

j. Bronchial artery

k. Subclavian artery

1. Descending thoracic aorta

4. Anomalous location of coronary ostium at improper sinus (which may involve joint origination or "single" coronary pattern)

a. RCA that arises from left anterior sinus, with anomalous course

(1) Posterior atrioventricular groove or retrocardiac

(2) Retroaortic

(3) Between aorta and pulmonary artery (intramural)

(4) Intraseptal

(5) Anterior to pulmonary outflow

(6) Posteroanterior interventricular groove (wraparound)

b. LAD that arises from right anterior sinus,

with anomalous course

(1) Between aorta and pulmonary artery (intramural)

(2) Intraseptal

(3) Anterior to pulmonary outflow

(4) Posteroanterior interventricular groove (wraparound)

c. Cx that arises from right anterior sinus, with anomalous course

(1) Posterior atrioventricular groove

(2) Retroaortic d. LCA that arises from right anterior sinus, with anomalous course

(1) Posterior atrioventricular groove

(2) Retroaortic

(3) Between aorta and pulmonary artery

(4) Intraseptal

(5) Anterior to pulmonary outflow

(6) Posteroanterior interventricular groove

\section{Single coronary artery}

B. Anomalies of intrinsic coronary arterial anatomy

1. Congenital ostial stenosis or atresia (LCA, LAD, RCA, Cx)

2. Coronary ostial dimple

3. Coronary ectasia or aneurysm

4. Absent coronary artery

5. Coronary hypoplasia

6. Intramural coronary artery (muscular bridge)

7. Subendocardial coronary course

8. Coronary crossing

9. Anomalous origination of posterior descending artery from the anterior descending branch or a septal penetrating branch

10. Split RCA

a. Proximal and distal PDs that both arise from RCA

b. Proximal PD that arises from RCA, distal PD that arises from $\mathrm{LAD}$

c. Parallel two PDs (arising from RCA, Cx) or "codominant"

\section{Split LAD}

a. LAD and first large septal branch

b. LAD, double (parallel LADs)

12. Ectopic origination of first septal branch
a. RCA
b. Right sinus
c. Diagonal
d. Ramus
e. $\mathrm{Cx}$

\section{Anomalies of coronary termination}

1. Inadequate arteriolar/capillary ramifications

2. Fistulas from RCA, LCA, or infundibular artery to:

a. Right ventricle

b. Right atrium

c. Coronary sinus

d. Superior vena cava

e. Pulmonary artery

f. Pulmonary vein

g. Left atrium

h. Left ventricle

i. Multiple, right and left ventricles

D. Anomalous anastomotic vessel left ventricular territories may be supplied by duplicated coronary arteries [4]. Duplications of LAD, Cx and RCA $[2,4]$ have all been reported. Dual LAD consists of one short and another long artery and is classified into 4 different subtypes. In the most common form (type I) the short and the long branches originate from the main LAD trunk. The shorter artery runs in the anterior interventricular sulcus and terminates abrupltly long before reaching the cardiac apex, while the longer one runs in the epicardial sur- face and returns in the interventricular sulcus in its distal third and then continues on the apex.

In the type II variety the long LAD courses over the anterior surface of the right ventricle. In type III the long LAD coronary has, at least partly, an intramyocardial (bridging) course. Unlike types I and II, the septal branches arise from long LAD and diagonals arise from the short LAD. Finally, in type IV the short LAD arises from left main while the long one arises anomalously from RCA and courses to the 
left side of the heart, anteriorly to the right ventricular outflow tract. In cases of double RCA, the two vessels may course together in the right atrioventricular groove and/or have separate courses with one of the two branches running on the epicardial surface of the right ventricle. Both vessels give rise to right ventricular branches and generally only one of the two branches gives off the posterior descending coronary artery [4].

3) Anomalies of coronary termination: this group comprises fistulas. A coronary fistula can involve both RCA and LCA, both ventricles, both atria and pulmonary artery and veins.

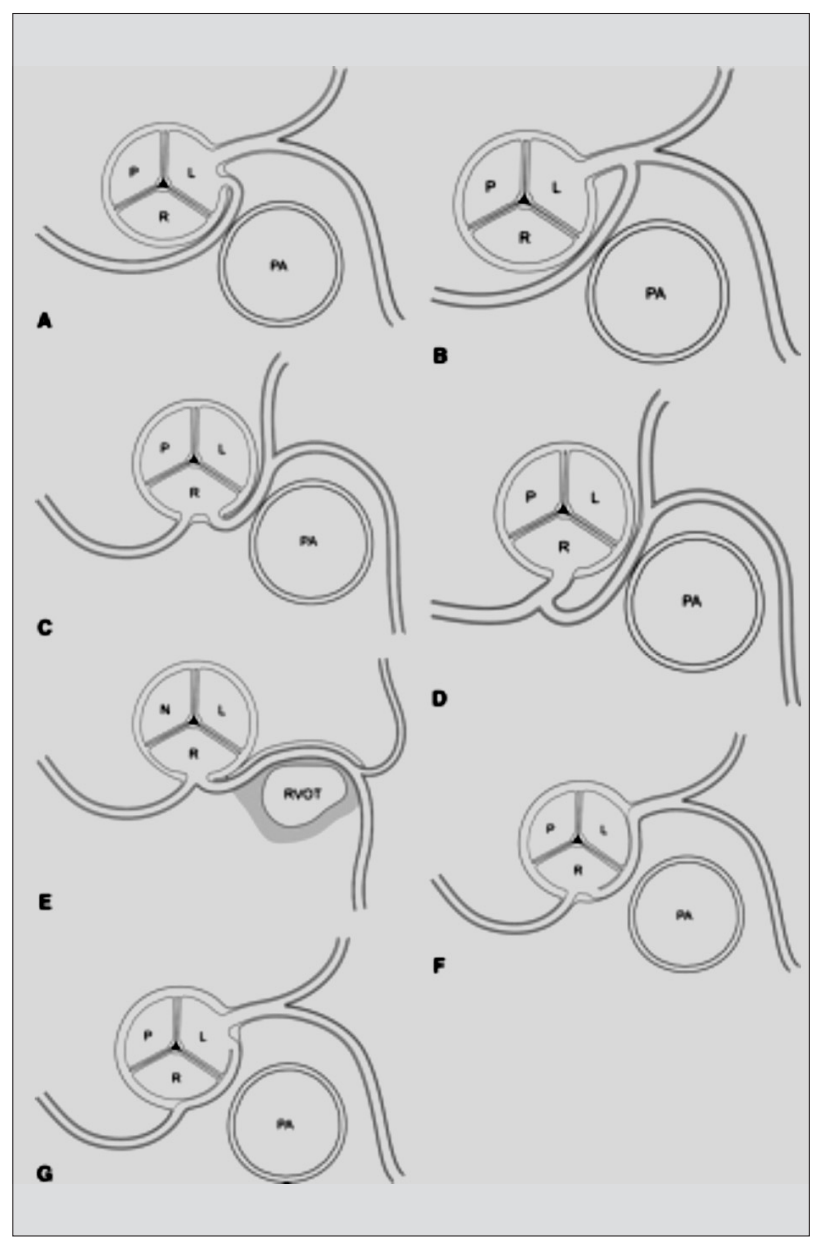

Figure 2. - High risk anomalous coronary artery patterns.

PA, pulmonary artery; P, posterior "non coronary" sinus of Valsalva; $\mathrm{L}$, left sinus of Valsalva; R, right sinus of Valsalva.

A. Anomalous right coronary artery (RCA) with separate ostium, arising from left coronary sinus (LCS), coursing interarterially, between the PA and aorta, without intramural course.

B. Anomalous RCA, arising from LMCA, and coursing interarterially between the PA and aorta, without intramural course.

C. Anomalous LCA arising with a separate ostium from right coronary sinus (RCS), coursing interarterially between the PA and aorta, without intramural course.

D. Anomalous LCA, arising from the proximal RCA, with a single ostium (shared with the RCA), and coursing interarterially between the PA and aorta, without intramural course.

E. Anomalous LCA with a single ostium (shared with RCA), arising from the RCS and coursing interarterially and intramuscularly between the PA and aorta.

F. Anomalous LCA originating from right sinus, with. intramural and interarterial course.

G. Anomalous RCA originating from left sinus, with. intramural and interarterial course.

\section{Coronary abnormalities in the catheterization laboratory}

Angelini et al. [1] examinated 1950 angiograms, finding the following prevalence of CAAs:

- Prevalence of CAAs in the general population: $5.64 \%$

- $\quad$ Split RCA: $1.23 \%$

- Ectopic RCA (left sinus): $1.13 \%$

- Fistulas: $0.87 \%$

- Absent left main coronary artery: $0.67 \%$

- Circumflex arising from right sinus: $0.67 \%$

In this section we describe some cineangiograms from our catheterization laboratory (Fig. 3-8).

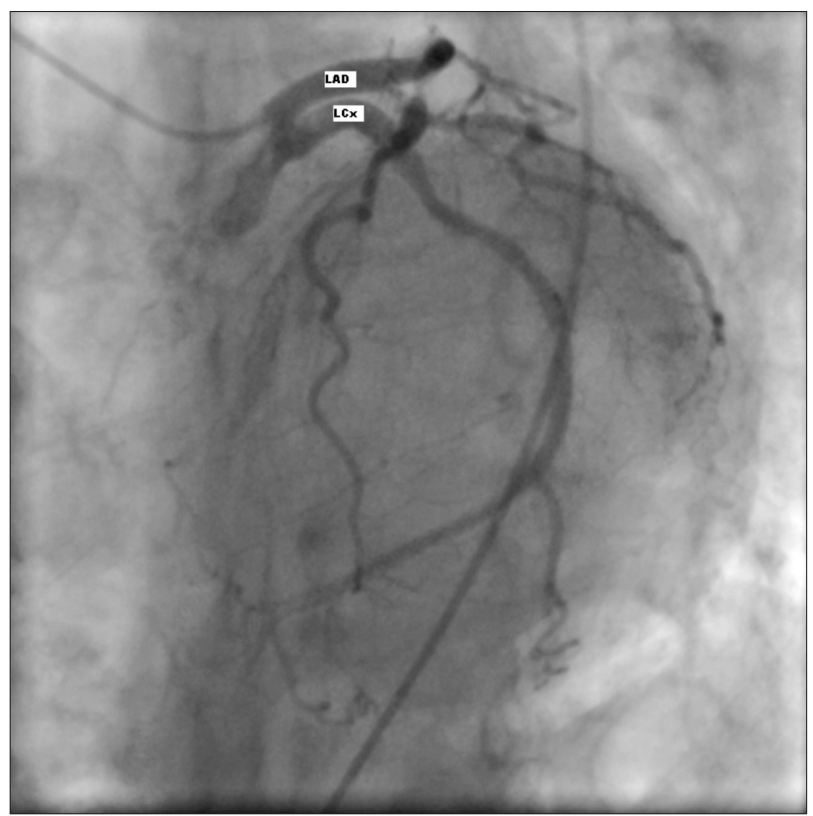

Figure 3. - Coronary angiography showing left main absence, with separate origin of left anterior descending (LAD) and left circumflex (LCx) coronary arteries from left coronary sinus. The absence of the left main with separate origin of the LAD and CX from left sinus has been described in roughly $1 \%$ of patients undergoing coronary angiography and is considered a normal variant.

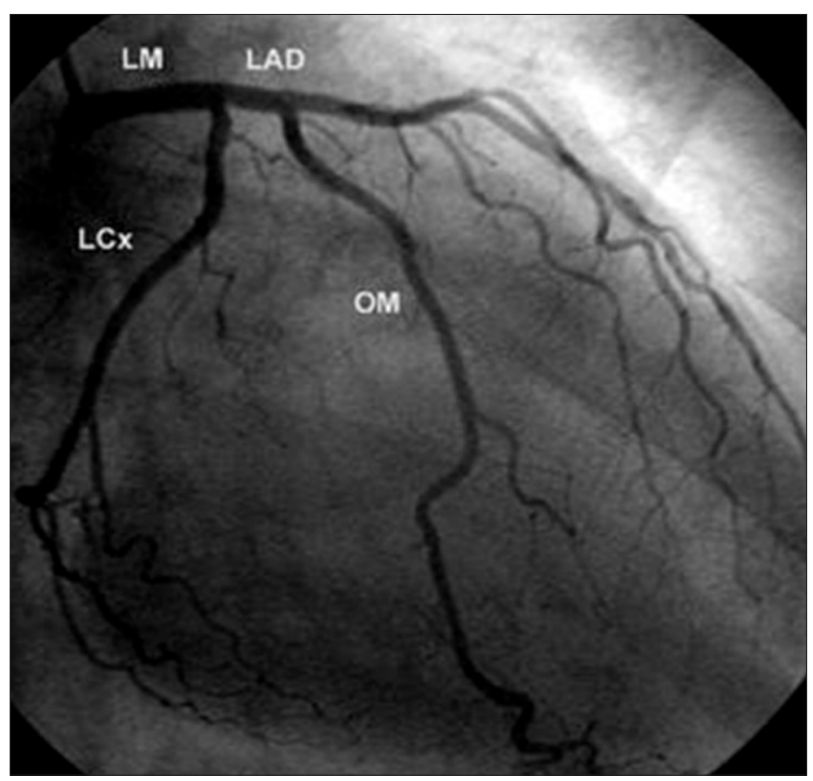

Figure 4. - Selective left coronary artery angiogram demonstrating anomalous origin of obtuse marginal (OM) coronary artery from proximal left anterior descending (LAD) coronary artery. LM= left main, LCX=left circumflex. 


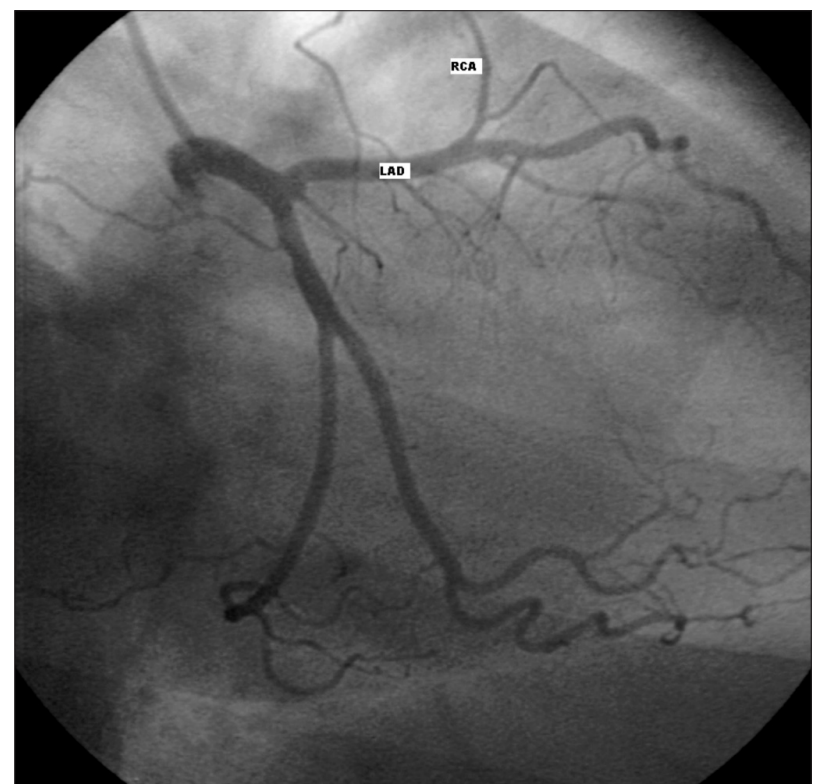

Figure 5. - Coronary angiogram showing anomalous origin of the RCA from the LAD with subsequent anterior course to the right atrioventricular groove.

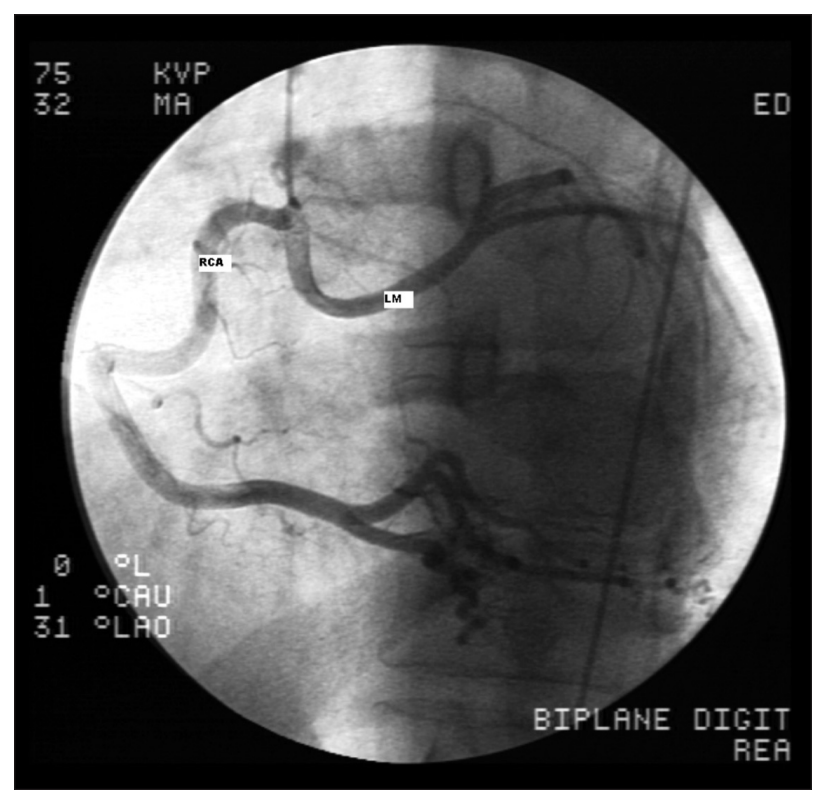

Figure 7. - Coronary angiography showing the anomalous orign of the left main (LM) coronary artery from proximal right coronary artery (RCA) with subsequent retroaortic (dorsal type D) course to the left side.

\section{Mechanisms of disease in CAAs}

To understand the clinical impact of CAAs, the fundamental challenge is to establish, for a particular type of CAA, a mechanism capable of interference with the coronary artery's function to provide blood flow to the dependent myocardium. Whereas some CAAs occasionally cause ischemia, others (eg, anomalous origin of the left coronary artery from the pulmonary artery and congenital coronary atresia) always cause ischemia, and yet others only predispose the patient to have a complication (clotting, spasm, or atherosclerotic buildup).

A pre-capillary fistula connecting a major coronary artery directly with a cardiac chamber, coronary sinus, superior vena cava or PA, is the most

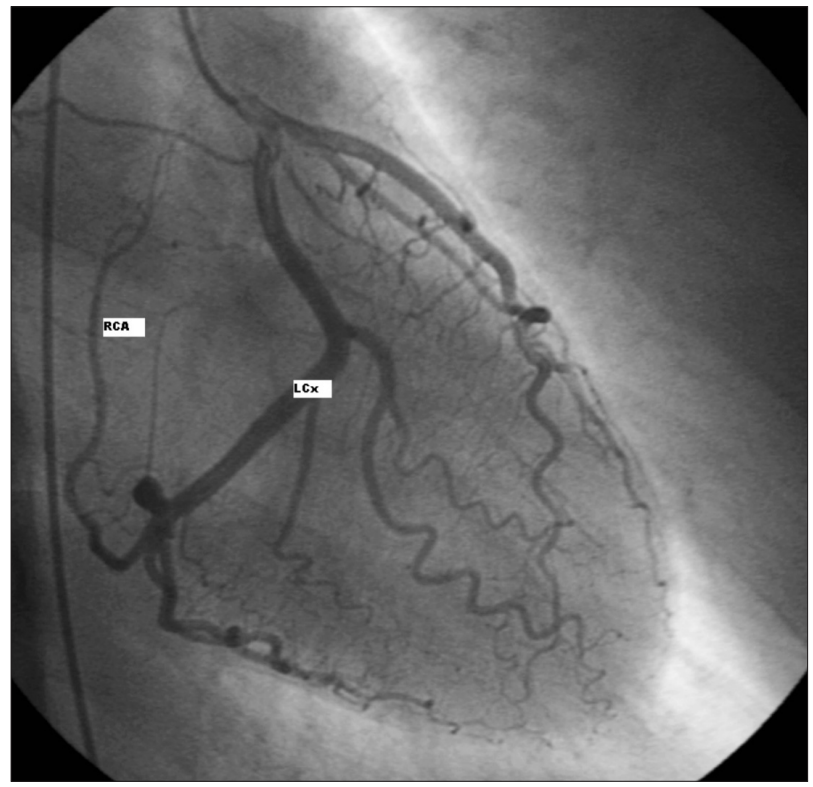

Figure 6. - Coronary angiography showing the origin of the RCA as continuation of the left circumflex (LCx) coronary artery.

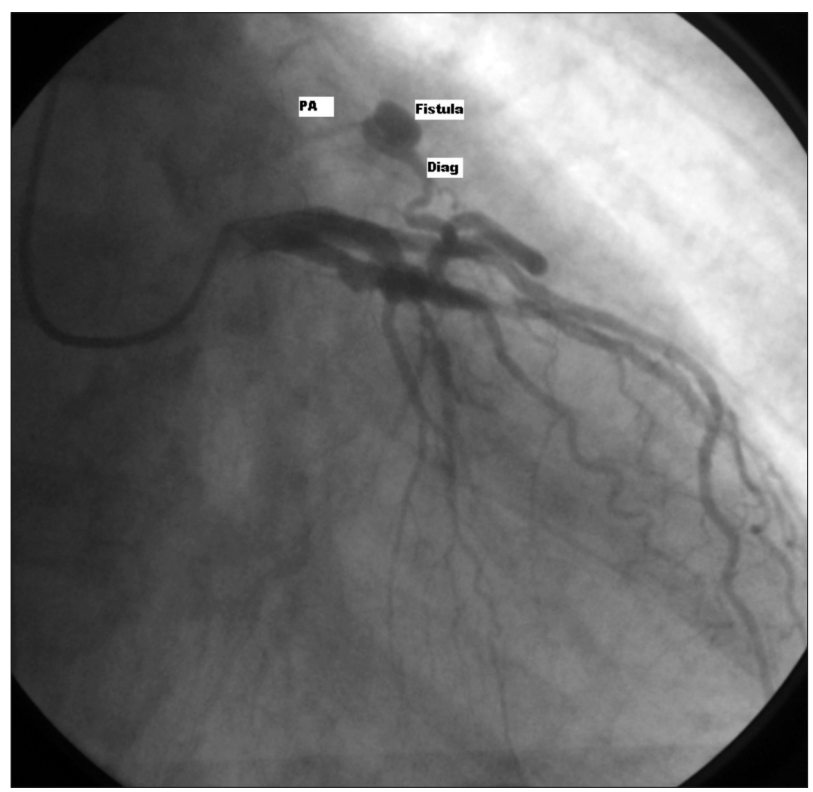

Figure 8. - Coronary fistula originating from a diagonal (diag) branch of the $\mathrm{LAD}$, with anomalous communication with the pulmonary artery (PA).

common form of hemodynamically significant coronary anomaly. Physiologically, a left-to-right shunt exists in such cases, except in the rare instances when the termination is in a left heart chamber. Approximately half of these patients are asymptomatic. The rest may develop congestive heart failure, subacute bacterial endocarditis, myocardial ischemia or infarction resulting from a "steal" phenomenon, or rupture of an aneurysmal fistula (5).

The anomalous origin of a coronary artery from the opposite sinus (ACAOS) has been recognized to have serious prognostic implications in young individuals [1, 6-7]. In cases of ectopic origination of a coronary artery, a severe prognosis is given by a specific abnormal course, called "interarterial", between the aorta and the pulmonary 
artery. Indeed, ACAOS has recently been observed, on intravascular ultrasound (IVUS) imaging, to consist of intramural proximal intussusception of the ectopic artery at the aortic-root wall [8]. Often, the intramural intussuscepted segment of the proximal ectopic artery is smaller in circumference than the more distal extramural vessel, so creating a situation of coronary hypoplasia. Arteries that arise congenitally inside the aortic media likely cannot grow normally either before or after birth. Moreover, the intramural segment is subjected to lateral compression, resulting in an ovoid (and not circular) cross section of the same segment, giving a smaller area than that possessed by a circle of the same circumference. This parameter can be quantified with the asymmetry ratio (the ratio of the smallest to the largest diameter in an IVUS cross section). Additionally, our group has observed that the smaller diameter is further compressed during each systole, as manifested by pulsatile behavior observed with IVUS during the cardiac cycle. This liability to undergo intermittent worsening is most likely related to changes in stroke volume (and pulsatility of the ascending aorta) and to tachycardia, which is a behavior that becomes manifest during IVUS imaging when an experimental pharmacological challenge simulates exercise conditions. When a carrier of ACAOS dies suddenly, in the absence of other lethal cardiovascular conditions, a low cardiac output and bradycardia or asystole typically occur early after extreme exercise, after which syncope and/or death ensues. Terminal ventricular fibrillation may also occur as a manifestation of critical ischemia or of reperfusion arrhythmia.

\section{Clinical repercussions of coronary artery anomalies}

CAAs are usually compatible with normal prenatal myocardial development and postnatal growth and function, even permitting intense athletic activity. Nevertheless, the anomaly sometimes leads to a pathological state, which usually originates suddenly and may have catastrophic consequences. A typical example of this situation is the anomalous origination of the left coronary artery from the opposite sinus (ACAOS) with intussusception of the proximal segment of the artery in the aortic wall (for details see text above).

CAAs have been implicated in chest pain, sudden death, cardiomyopathy, syncope, dyspnea, ventricular fibrillation and myocardial infarction. Rarely, they have been related to reproducible effort angina, as seen in obstructive coronary disease.

CAAs might have clinical consequences other than those strictly related to myocardial ischemia; these consequences might include volume overload (in cases of large coronary fistulas), aortic-root distortion (in cases of very large coronary fistulas or aneurysms), bacterial endocarditis, complications during aortic valve surgery or coronary angioplasty, and misdiagnosis (as in many cases of "missing", coronary arteries).

\section{Therapeutic options}

While many CAAa seems to be only anatomical curiosities without clinical repercussions (but often representing a proof of patience for the physician performing coronary angiography), others may have serious consequences, requiring medical or interventional treatment.

Symptomatic carriers of ACAOS have 3 treatment options: medical therapy/observation, coronary angioplasty with stenting, or surgical repair. Despite the limitations of our knowledge of such anomalies, intervention may be justified in some cases to prevent sudden cardiac death and improve the quality of life. Medical treatment (essentially with beta-blockers) is probably as effective as restriction of activity (avoidance of extreme exertion) in these patients [9]. PCI of the obstructed proximal intramural segment of a patient with right-ACAOS is technically feasible [10], and is probably justifiable in the presence of disabling symptoms and/or a high risk of sudden death, area stenosis more severe than $50 \%$ with respect to the distal normal vessel on IVUS, a large dependent myocardial territory (more than a third of the total), and inducible ischemia, as documented by a stress test. Drug-eluting stents offer the best probability to avoid restenosis, but definitive data need to be collected regarding this offlabel use of stents. Surgical treatment of ACAOS has been performed in large series of patients for several years. Surgical correction, which is especially recommended for left-ACAOS that involves a large territory at risk, may consist of: 1) direct reimplantation of the ectopic artery at the aortic root (a technically difficult and unreliable approach);2) unroofing of the intramural coronary segment, from the ostium to the exit point, off the aortic wall; or 3) osteoplasty, which creates a new ostium at the end of the ectopic artery's intramural segment [11]. Symptomatic carriers of a left coronary artery originating from the pulmonary artery (giving rise to large fistulas with volume overload and ischemia) can be treated surgically, with reimplantation of the aberrant coronary ostium on the aortic root.

Myocardial bridge (an entity that is familiar to every interventional cardiologist) with involvement of a large myocardial territory and complete systolic obliteration of the vessel, can be related to malignant ventricular arrhythmias and sudden death, requiring surgical resection.

\section{Considerations and conclusions}

The challenge presented by coronary anomalies is understanding the variability of their functional repercussions. Some anomalies (including most of those in unselected necropsy series) seem to be only curiosities. Other anomalies are potentially fatal. Still, cardiologists can only guess at possible pathophysiological mechanisms for the latter while lacking the information necessary to make sound recommendations. Cardiologists must transcend their current predominant approach, characterized by no more than the periodic publication of dramatic case reports. Our knowledge about the clinical expression of coronary anomalies could be 
greatly enhanced by a multicenter database capable of prospectively collecting information about the following fundamental issues: (1) the incidence of each anomaly, as an anatomic entity, in unselected general populations, based on strict, prospectively agreed-on diagnostic criteria; (2) the type and incidence of clinical manifestations associated with each type of anomaly and the specific features of each instance; (3) the detailed clinical history of each patient with a given anomaly who experiences a clinical event, with particular attention to objective documentation of the event itself and the relevant clinical circumstances (exercise, dehydration, smoking, drugs, etc); and (4) the treatment (behavorial, medical, or surgical) used for a given patient with a coronary anomaly, whether identified during life or after death. Living patients should undergo long-term observation to clarify the anomaly's natural history and the influence of any empirical intervention. Using powerful databases (ideally under the auspices of a large professional organization), such a network of experts should implement coordinated, pre-established protocols for clinical investigations. These experts should also support educational efforts, publish periodic documents, and foster pertinent discussions by holding regular meetings. In conclusion, if $19 \%$ of sudden deaths in young athletes are due to coronary anomalies, and if most anomalies are harmless but the rest require aggressive intervention, we owe our patients and colleagues a precise, objective source of information that can lead to sound recommendations and safe treatment.

\section{Riassunto}

Le anomalie coronariche costituiscono uno degli argomenti meno chiari della cardiologia e colpiscono circa l'1\% della popolazione generale. Mentre alcune anomalie sembrano essere solo delle curiosità anatomiche, altre possono rivelarsi fatali. Questo lavoro passa in rassegna le principali caratteristiche anatomiche delle più frequenti anomalie coronariche e si focalizza sui meccanismi fisiopatologici mediante $i$ quali le suddette anomalie possono causare una condizione patologica. L'ultima sezione descrive le opzioni terapeutiche di questi disordini congeniti.

\section{ABBREVIATIONS}

CAAs: Coronary artery anomalies

LAD: left anterior descending coronary artery

Cx: circumflex coronary artery

RCA: right coronary artery

PA: pulmonary artery

\section{References}

1. Angelini P, Villason S, Chan AV, Diez JG. Normal and anomalous coronary arteries in humans. In: Angelini P, ed. Coronary Artery Anomalies: A Comprehensive Approach. Philadelphia: Lippincott Williams \& Wilkins; 1999: 27-150.

2. Angelini P. Coronary Artery Anomalies: An Entity in Search of an Identity. Circulation 2007; 115; 1296-1305.

3. Corrado D, Thiene G, Cocco P, Frescura C. Non-atherosclerotic coronary artery disease and sudden death in the young. Br Heart J 1992; 68: 601-607.

4. Misuraca L, Rutigliano D, Pestrichella V, Contegiacomo G, Balbarini A. A very rare congenital abnormality: double right coronary artery. A case report. $J$ Cardiovasc Med (Hagerstown) 2009 Aug; 10(8): 643-5

5. Angelini P, Velasco JA, Flamm S. Coronary Anomalies: Incidence, Pathophysiology, and Clinical Relevance. Circulation 2002; 105; 2449-2454

6. Alexander RW, Griffith GC. Anomalies of the coronary arteries and their clinical significance. Circulation 1956; 14: 800-805.

7. Bharati S, Lev M. The Pathology of Congenital Heart Disease. Armonk, NY: Futura Publishing Company; 1996.

8. Angelini P, Velasco JA, Ott D, Khoshnevis GR. Anomalous coronary artery arising from the opposite sinus: descriptive features and pathophysiologic mechanisms, as documented by intravascular ultrasonography. J Invasive Cardiol 2003; 15: 507-514.

9. Maron BJ, Zipes DP. Introduction: eligibility recommendations for competitive athletes with cardiovascular abnormalities-general considerations. J Am Coll Cardiol 2005; 45: $1318-1321$.

10. Hariharan R, Kacere RD, Angelini P. Can stent-angioplasty be a valid alternative to surgery when revascularization is indicated for anomalous origination of a coronary artery from the opposite sinus? Tex Heart Inst $J$ 2002; 29: 308 -313.

11. Mustafa I, Gula G, Radley-Smith R, Durrer S, Yacoub M. Anomalous origin of the left coronary artery from the anterior aortic sinus: a potential cause of sudden death: anatomic characterization and surgical treatment. J Thorac Cardiovasc Surg 1981; 82: 297-300. 